\title{
A Review on Mobile Cloud Computing and Issues in it
}

\author{
Saqib Hakak \\ MS - Computer Engineering \\ International Islamic University \\ Malaysia
}

\author{
Suhaimi A Latif \\ Asst. Professor \\ International Islamic University \\ Malaysia
}

\author{
Gulshan Amin \\ M.Tech \\ S.I.T.M \\ India
}

\begin{abstract}
Cloud computing is one of the emerging technology in this modern era. Some years back, this concept was related to only simple desktop computers or personal computers and static data centers and so on but now research is going on to implement this concept to mobiles also. So, in this paper, the concept of cloud computing as a general is given, followed by concept of mobile computing along with the general issues which are creating hindrances in completing this technology.
\end{abstract}

\section{General Terms}

Review of Mobile Cloud Computing, Issues in mobile cloud computing.

\section{Keywords}

Mobile cloud computing (MCC), Issues in Mobile cloud computing, cloud computing and Mobile computing.

\section{INTRODUCTION}

Cloud computing is one of the most recently emerged technology which offers resource on demand facility. This technology allows much more efficient computation by centralizing storage, memory, and processing and band width and due to these factors, this technology will have a great impact once it will be complete in all aspects. According to statistics by a famous global consulting company, in near future, the cloud computing will be among top ten trends in terms of technology[1] which implies that, most organizations would prefer this technology as it is easy to use and very economical also. Currently, researches are going on, whether this technology can be implemented to mobile phones also. And this concept of combination of technologies which involves merging of cloud computing concept with mobile computing gives rise to a new concept, which the researchers have named Mobile cloud computing (MCC).And currently many such applications have also been developed like Google maps or navigation systems for mobiles or application from Apple known as Mobile Me, similarly, there are other few applications from different vendors such as Moto blur, voice search and so on [2]. Similarly, in another research, which was conducted by juniper, statistics have shown that by the end of year 2014 , the applications based on mobile clouding concept will rise by almost $88 \%$ approximately and from these statistics, one can imagine once this technology will be get complete ,how much impact will it have on a society. Although, there are few applications as mentioned above based on this concept, but there are many challenges, which need to be overcome to implement this technology fully .One of the major problem is how to combine these two technologies in a single technology as there are many problems associated with that like limitations of mobile phones in terms of battery life, limited storage, bandwidth issue and so on. Similarly, there is architectural issue also. Thus, in order to implement this technology fully, it is must to have a clear picture of exactly, what is mobile computing and what are the major issues in merging these technologies in one technology. Thus, in this paper, the concept of mobile computing is given along with its major issues.

The organization of paper is as follows: Section 2 is related to concept of mobile cloud computing. Section 3 is related to major issues in mobile cloud computing (MCC) and finally in section 4 , conclusion is given.

\section{Concept of Mobile Cloud Computing}

Since the last few years, the smart phones has brought a revolution to the current age of modern scientific era .Besides ,being portable, it has not only made loved ones to stay connected irrespective of the longer distances through voice calls but now, with the advent of $3 \mathrm{G}$ technology, one can also have an advantage of video calls also .Similarly ,there are various other technologies like Gprs ,Edge, wcdma and so on ,which has made this smart phone like a smart computer through which user can browse, make calls, can avail gps facility and so on. Mobile cloud computing is actually a term which is made up of two terms or derived from two terms namely cloud computing and mobile computing. So, before getting full understanding of mobile cloud computing, it is must to have clear understanding of cloud computing and mobile computing.

\subsubsection{Cloud computing}

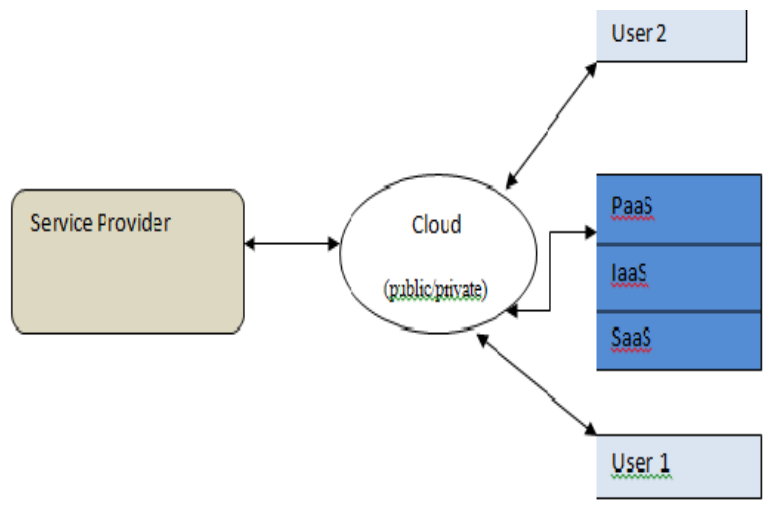

Fig 1: Concept of cloud computing 
The concept of cloud computing is quite simple .It is that type of computing which relies on sharing of computer resources rather than having personal devices or servers to handle applications [3].It is completely internet dependent technology and each client is assigned its own cloud with the help of which that client can use different services like services of servers, storage, manage applications and so on as per pay service basis. Here, the word cloud is used as metaphor for the internet [3].In the fig 1 , the concept is simple, two users i.e. user one and user 2 are using the cloud and cloud can provide any service to these users, which are required by the users like Saas, Paas or Iaas service. The cloud, in turn is managed by any third party.

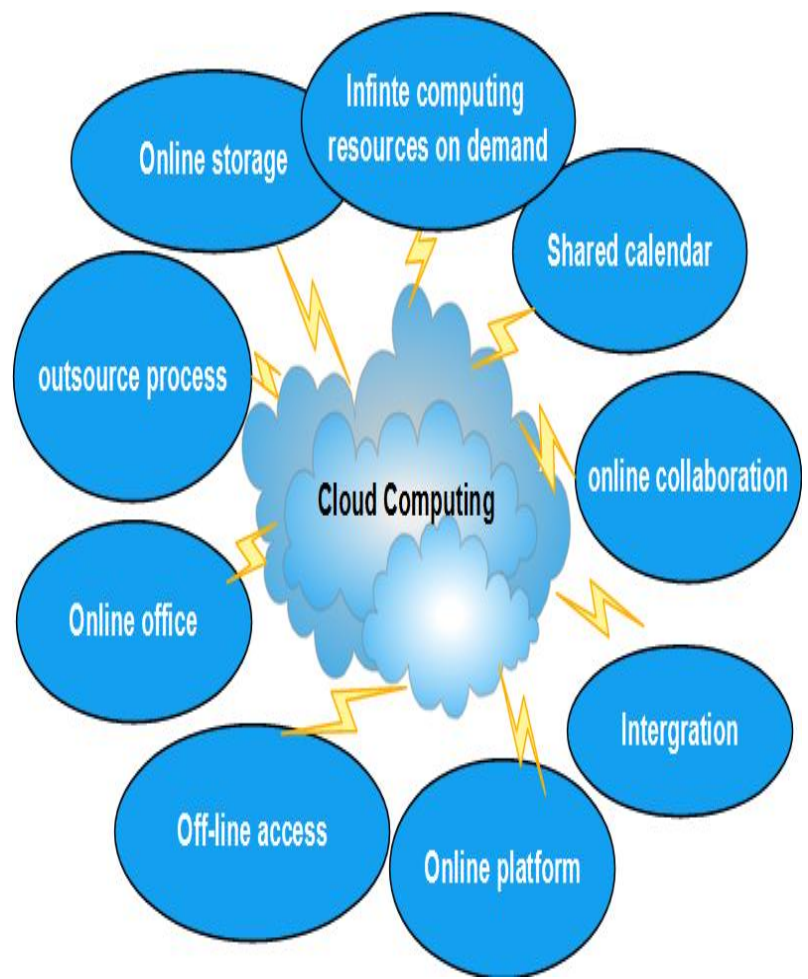

Fig 2: Services of cloud computing

Thus, in simple terms, cloud computing can be defined as that technology in which end users make use of cloud through internet and this cloud can be maintained by any third party or any other company and this cloud provides three basic services like Paas, Iaas and Saas. Paas, which stands for platform as a service is utilized by web developers for the application development and deployment platform purposes over the web. All applications with the help of programming languages and tools are developed here. And this service offered by cloud computing is very useful for the developers as they can use the equipment's without requiring to have those equipment's physically and can develop quality programs and then transfer to the end users via internet, thus reducing both cost as well as complexity. In Iaas, which stands for infrastructure as a service, the users acquire computing resources like memory, processing power and storage from IAAS provider and make use of resources to run and deploy their applications [4]. In Saas, which stands for software as a service, the users simply view the software developed by others through web browser and offer a service to users to buy it. In other words, we can say that, in SAAS, readymade software is developed by some third party and then that particular software is made available to users for using that software.

\subsubsection{Mobile Computing}

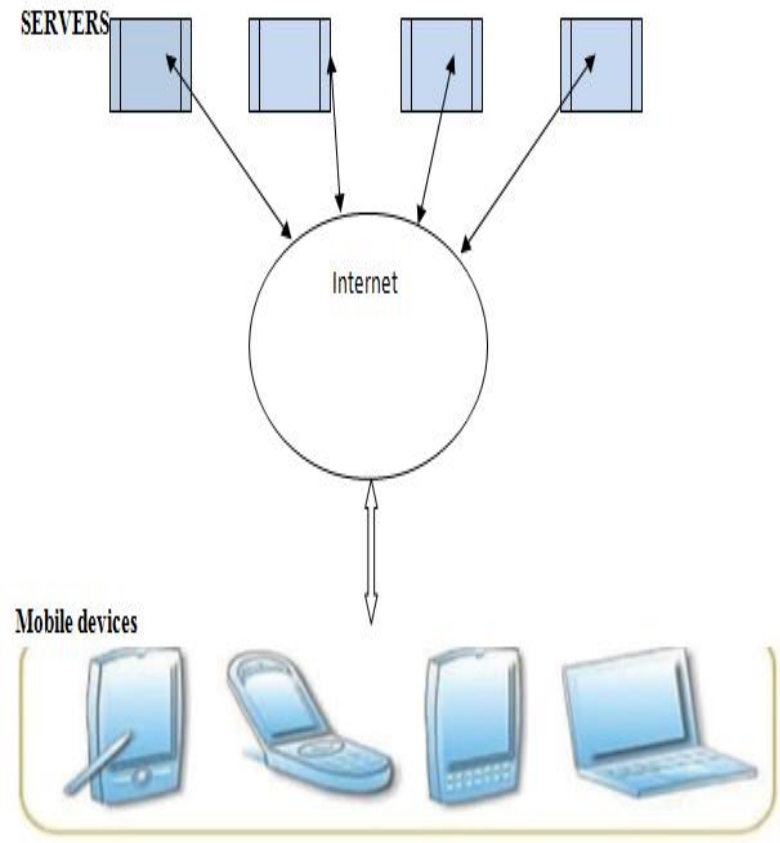

Fig 3: Concept of Mobile computing

By the term mobile computing, we simply mean any device which is movable with computing capability. Mobile phones are best examples of mobile computing. In this modern era, almost $90 \%$ population does use mobile phones and these figures will rise. But, with these mobile phones, one can avail the internet facility also, despite his location but within the area of the service provider. So, by mobile computing, we can say any device, which is capable of letting users to avail the services of using internet or any other similar service while on the go, meaning users can avail the services while walking, while travelling to other place and so on but provided, the user is within the area of service provider. There are three major components on which mobile computing are based and these components are: hardware, software and communication link. Hardware part implies physical portable devices like mobile phones, laptops .their components and so on. Software part implies, their respective operating systems, mobile applications like antivirus, games and so on. And the last part implies infrastructure or networks which provide services like internet service, voice call service and so on to these mobile appliances and this component remains transparent to end users [2].

Although , the mobile computing has made the life of people a lot easier but it suffers from some limitations .Firstly, since mobile user are always on the move , the mobile network service provider may have weak signal strength in any particular area and in that case ,the mobile user may not get the proper service. Similarly, mobile signals are prone to interference and that issue also creates hindrance in providing quality service. The bandwidth issue is also one of the main issue in mobile computing. Similar such issues are limited storage, delay in handoff, low capability issue and so on.

\subsection{Mobile Cloud Computing}

Now, from the above discussion, we have clear picture of what is mobile computing and cloud computing. The combination of both these technologies is known as mobile cloud computing (MCC). Day by day, the hardware part along 
with software capability is getting more solid and sophisticated in mobile phones. Now, the phones are coming with Giga hertz processors and 1 GB rams due to which their computing capability is also getting faster and stronger. Similarly, the operating systems like android series have made it further faster with support to many mobile applications. The latest version of android which is Jelly bean has many features as compared to its predecessor Ice cream sandwich. Today, many mobile applications are dedicated to navigations systems, maps, games, simulators, browsers, media players and much more. Also, according to Eric ,CEO of Google ,in his interview has stated that mobile phones based on cloud computing service, will become more complicated and will be called super computers very soon [5].

In mobile computing, the concept is similar as in cloud computing i.e. the user's request access for any particular service from cloud through internet which provides the dedicated service. However, here instead of fixed user as is in cloud computing, the users are mobile i.e., they can be anywhere like walking, in car, in office and so on. Also, in MCC, the intensive processing and storage has been transferred to a cloud and this has greatly reduced processing and storage load from mobile devices. And this has changed the whole scenario of deployment and running of mobile applications. As shown in fig 4 , the users are mobile which can be mobile phone users, tablet users and so on and the major services like processing, support, storage etc. have been transferred to cloud and this has greatly reduced the load from mobile phones and due to MCC, now even low budget phone can also give users required services. Thus, any mobile user can avail these services through a cloud via internet connection which can be $3 \mathrm{G}$ connection, wifi or gprs and so on and that particular cloud will be serving their requests.

\section{Issues in Mobile cloud computing}

The main objective and aim of mobile cloud computing technology is to provide any desired service which a mobile user wants to avail irrespective of location or place. But, there are many limitations which are creating hurdles in making this technology complete. According to chetan ,the most striking challenge comes from the characteristics of mobile devices and limitations which makes application designing ,programming and deploying on mobile and distributed devices more complicated than fixed cloud device [6].Thus , the factors like connectivity, support of cloud to mobile user etc are some factors which are affecting the implementation of Mobile cloud computing. It is not possible to jot down each issue in a single paper, thus below are some of the major factors which are affecting this technology.
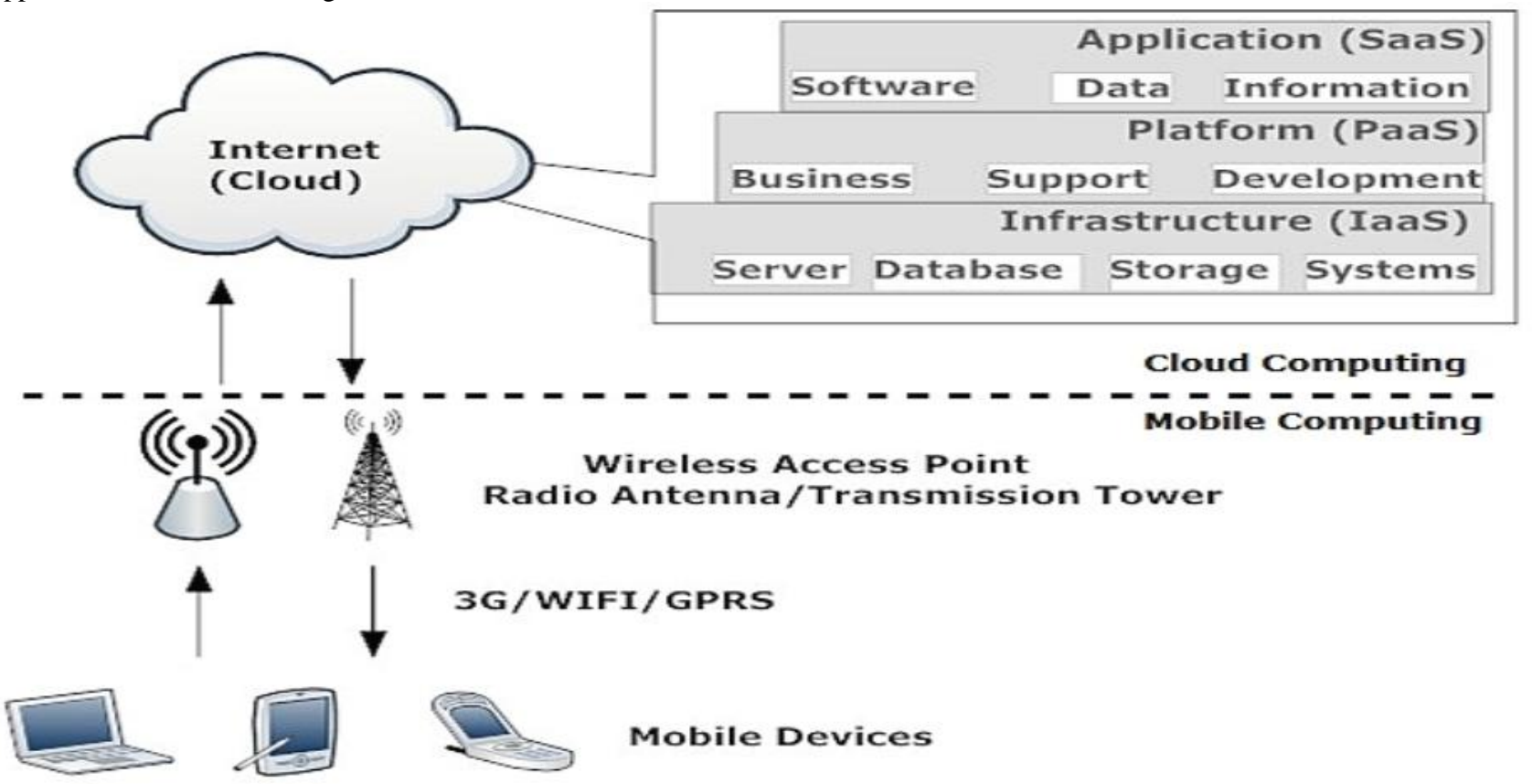

Mobile Devices

Fig 4: Concept of Mobile Cloud Computing [2]

\section{Physical specifications of Mobile devices:}

Although, within these years, some rapid changes have come in terms of improvement in the hardware and software specifications of mobile phones like increased storage capacity, large Amoled displays, powerful processors but still not all devices are up to the mark. These devices are in no match to fixed desktops or laptops which are having much more and many times stronger processors and storage capabilities. Besides, the biggest limitation is very short span of battery life. On an average, if a user uses 6 hours of internet service on a mobile phone, almost whole battery will get drained within those hours and this issue is not in fixed desktops, where a user has uninterrupted power supply. So, shorter span of battery and limited processing capability is one of the limitation.

\section{Inconsistent Bandwidth:}

In case of wired networks, like LAN, the bandwidth is fixed and users enjoy the high link speed without any interruption. However, in case of mobile devices, which usually use wireless networks, this is not a case. There may be a congested network sometimes, which will affect the wireless service. Similarly, bad weather, interference, delay in hand off , wireless site, heavy load of users on a particular wireless site are some other factors which will affect the performance of providing quality of service to end users via mobile cloud 
computing. Normally, in wired networks, the latency delay is $50 \mathrm{~ms}$ which is $200 \mathrm{~ms}$ in case of wireless network.

\section{High computing Applications:}

Some applications may require massive processing power and storage, which cannot be deployed in mobile devices due to limited resources. Under such circumstances, we have to divide applications in such a way so that the complex part is done by a cloud and a simpler task is left to mobile device [2].

\section{Delay in input/output from user to cloud:}

As mentioned above, wireless networks are prone to bandwidth issues, congestion issues and so on, as compared to wired networks, in case of mobile cloud computing, which will be mostly depending on wireless networks, the cloud may take time in accepting and providing the required services to users quickly.

\section{Conclusion}

Mobile cloud computing is one of the new and major emerging technology in this era of modern and scientific age as it provides many services like saas, iaas, paas in very easy and efficient way to mobile users. However, in order to make this under process technology perfect and complete, the most important issues related to its security, implementation, providing quality of service must be addressed and resolved once for all as no one can compromise with these basic services of his/her data. Although, researches are going on in the same domain but it needs more efforts. In this paper, concept of mobile cloud computing and major issues in implementing this technology is addressed. There is lot of future work to be done in this very domain. Initial emphasis can be laid to improve processing performance and battery performance while executing large and memory consuming applications.

\section{ACKNOWLEDGMENTS}

Our thanks to the Professors, experts and other faculty members who provided useful resources and background to complete this research paper

\section{REFERENCES}

[1] M. Cooney. (2011, Oct) Gartner: The top 10 strategic technology trends for 2012. [Online]. Available: http://www.networkworld.com/news/2011/101811gartner-technology-trends-252100.html

[2] webopedia

[3] Han Qi, Abdullah Gani - Research on Mobile Cloud Computing: Review, Trend and Perspectives

[4] Ilango Sriram, Ali Khajeh-Hosseini ,Research Agenda in cloud technologies

[5] B. Marrapese. (2010, Dec.) Google ceo: a few years later, themobile phone becomes a super computer [Online].Available:http://www.itnewsblog.com/it/21320. html

[6] S. Chetan, G. Kumar, K. Dinesh, K. Mathew, and M. Abhimanyu, Cloud computing for mobile world," available at chetan. ueuo. com

[7] E. Marinelli, - 2009 ,Hyrax: cloud computing on mobile devices using MapReduce,\| Master thesis, Carnegie Mellon University 